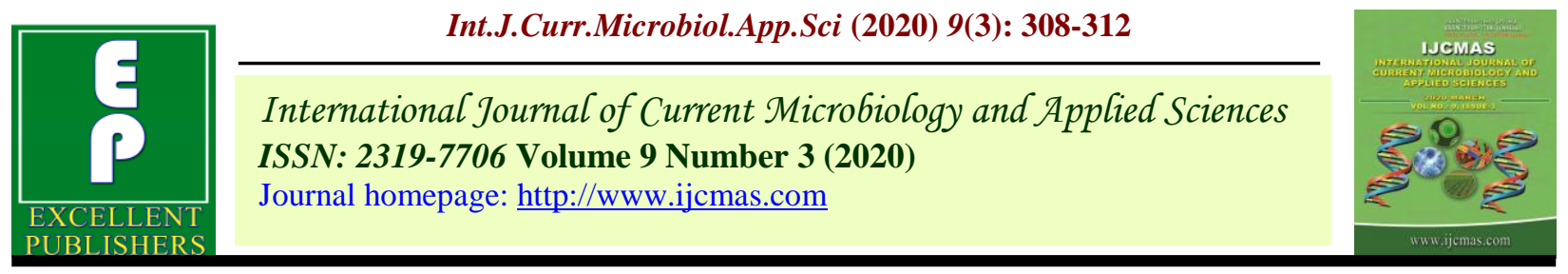

\title{
Diagnostic Role of Polymerase Chain Reaction and Inadequacy of Mycobacterium Growth Indicator Tube culture in Tuberculous Pleuritis
}

\author{
Gandhi Kandhakumari ${ }^{1 *}$ and Selvaraj Stephen ${ }^{2}$ \\ ${ }^{1}$ Kanti Devi Medical College and Research Centre, Mathura, Uttar Pradesh-281406, India \\ ${ }^{2}$ Department of Microbiology, Mahatma Gandhi Medical College \& Research Institute, SBV \\ University, Pillaiyarkuppam, Pondicherry - 607403, India \\ *Corresponding author
}

\begin{tabular}{l} 
K e y w o r d s \\
PCR, \\
$\begin{array}{l}\text { Mycobacterium } \\
\text { tuberculosis, } \\
\text { Pleural fluid, LJ, } \\
\text { IS6110 }\end{array}$ \\
Article Info \\
$\begin{array}{l}\text { Accepted: } \\
\text { 05 February } 2020 \\
\text { Available Online: } \\
10 \text { March } 2020\end{array}$ \\
\hline
\end{tabular}

\section{A B S T R A C T}

Diagnosis and management of patients with tuberculous pleuritis needs a rapid and sensitive diagnostic tool. Our aim was to diagnose cases of tuberculous pleuritis from patients employing ZN smear, culture (LJ \& MGIT) and to compare with PCR. Pleural fluid collected from 36 patients with clinical and/or radiological suspicion of TB pleuritis was included in this study. Pleural fluid collected from nine non-tuberculous pleuritis cases was included as negative controls. Pleural fluid samples were analyzed for ZN smear, LJ and automated MGIT culture and results compared with MTB PCR targeting IS6110 region. Positivity for smear, LJ, MGIT and PCR individually were $5.6 \%, 2.8 \%, 16.7 \%$ and $25 \%$ respectively. Combination of all above techniques together increased the percentage positivity to $30.6 \%$. Negative controls were negative by all procedures. In the context of paucibacillary specimens like pleural fluid, smear microscopy and LJ are less sensitive. MGIT has moderate sensitivity and rapidity compared to LJ. PCR is rapid and highly sensitive, but needs interpretation of PCR reports with "gold standard" culture and clinical history to arrive at an early and accurate diagnosis.

\section{Introduction}

India, a developing country with high tuberculosis endemicity accounts for highest incident cases of $27 \%$ among the total cases reported worldwide (WHO, 2018). Pleural tuberculosis ranks second next to tuberculous lymphadenitis and India accounts for 30-80\% of cases. Though pleural biopsy is reported as the optimum specimen for definitive diagnosis of tuberculous pleural effusion, pleural fluid is commonly collected for diagnostic purpose (SK Sharma et al., 2004; P James et al., 2010). Because pleural fluid tends to be paucibacillary it turns out to be negative in 80 to $90 \%$ cases making conventional microbiological techniques like smear and culture least sensitive. But molecular techniques which detect and amplify specific regions of $M$. tuberculosis 
genome are considered more sensitive when compared to conventional diagnostic modalities (Sharma et al., 2004; Makeshkumar et al., 2014; Siddiqui et al., 2013; Shital et al., 2014; Kandhakumari et al., 2015). Hence in this study Ziehl-Neelsen smear (ZN) microscopy, culture on Lowenstein-Jensen media (LJ) and Mycobacterium Growth Indicator Tube 960 (MGIT 960) were carried out and compared with that of Polymerase Chain Reaction (PCR) targeting IS6110 region for diagnosis of tuberculous pleuritis.

\section{Materials and Methods}

This work was approved by Institutional Human Ethical Committee and the work was carried out in accordance with the Declaration of Helsinki. Written informed consent was obtained from patients before collection of clinical samples. Patients with clinical/radiological suspicion of TB pleuritis were included. Patients suspected for tuberculous pleuritis with pulmonary tuberculosis, sputum positive cases and those treated with ATT (Anti-tuberculous treatment) drugs were excluded. With sterile precautions, pleural fluid from 36 patients attending our hospital with clinical and/or radiological suspicion of TB pleuritis between June 2011 and October, 2014 was included in this study. Pleural fluid from nine non-TB patients (five cases of pneumonia and four cases of Chronic Obstructive Pulmonary Disease) were included as negative controls. The specimens were centrifuged, and deposits stained by ZN. Inoculation of the deposit was made on LJ and MGIT 960 tubes and incubated up to eight and six weeks respectively. Identification of $\mathrm{M}$. tuberculosis complex (MTBC) recovered in LJ and MGIT was confirmed by standard biochemical tests and a rapid immunochromatography based SD Bioline MPT64Ag detection kit (G Kandhakumari et al., 2015).

\section{Nested Polymerase chain reaction (nPCR):}

DNA from pleural fluid was extracted as per the guidelines of the manufacturer (Amplification Reagent Set for Mycobacterium tuberculosis, Bangalore Genei, Bangalore, India) for nPCR. This is single-tube nested PCR with two - step sequential assay. In the first step, 220 bp Insertion sequence (IS6110) was amplified and in second step nested primers were added to the amplicon to further amplify the $123 \mathrm{bp}$ region. Gel electrophoresis was carried out and amplified products were observed using gel documentation system (BIO-RAD, Hercules, CA, USA). Positive and negative controls were included and monitored to avoid false positivity and carry over contamination.

\section{Statistical analysis}

Sensitivity, specificity, positive predictive value and negative predictive value of different diagnostic methods were determined and compared using Chi-square test with or without Yate's correction. $\mathrm{P}$ values $<0.05$ were considered statistically significant.

\section{Results and Discussion}

Among 36 pleural fluids processed, 5.6\% was positive by smear, $2.8 \%$ by growth on $\mathrm{LJ}$ and $16.7 \%$ by growth in MGIT (Table. 1). All six isolates were $M$. tuberculosis and none belonged to non tuberculous Mycobacteria (NTM) group. Two pleural fluid samples which were positive by smear did not grow M.tuberculosis on LJ, but were isolated in MGIT 960 system. Among 34 smear negative pleuritis patients, four isolates of M.tuberculosis (11.08\%) were recovered from MGIT, and one from LJ (2.8\%). PCR alone yielded $25 \%$ positivity. All techniques together increased the positivity to $30.6 \%$. Among the 34 smear negative samples, only $2.9 \%$ were positive by LJ, but $11.08 \%$ by 
MGIT and $23.5 \%$ by PCR (Table-1). All negative control samples were negative by all techniques. Keeping culture as the gold standard, the sensitivity specificity, PPV \&
NPV for ZN smear was $60 \%, 100 \%, 100 \%$ and $86 \%$ whereas for PCR it was $66 \%, 83 \%$, $44 \%$ and $92 \%$ respectively.

Table.1 Comparison of PCR results with conventional techniques

\begin{tabular}{|c|c|c|c|c|c|c|}
\hline S & Smear & LJ & MGIT & PCR & $\begin{array}{l}\text { No. of } \\
\text { cases }\end{array}$ & $\begin{array}{l}\text { Percentage } \\
\text { positivity }\end{array}$ \\
\hline $\mathbf{1}$ & + & - & + & + & 1 & 2.8 \\
\hline $\mathbf{2}$ & + & - & + & - & 1 & 2.8 \\
\hline $\mathbf{3}$ & - & + & + & + & 1 & 2.8 \\
\hline $\mathbf{4}$ & - & - & + & + & 2 & 5.6 \\
\hline $\mathbf{5}$ & - & - & + & - & 1 & 2.8 \\
\hline $\mathbf{6}$ & - & - & - & + & 5 & 13.9 \\
\hline $\mathbf{7}$ & - & - & - & - & 25 & 69.4 \\
\hline Total positive & 2 & 1 & 6 & 9 & 36 & 100 \\
\hline
\end{tabular}

Diagnosis of TB pleuritis needs high index of clinical suspicion and appropriate specimen for effective diagnosis. TB pleuritis mainly occurs as allergic reaction to tuberculoprotein and hence the bacilli load is very low in pleural fluid. Though pleural biopsy is the preferred specimen, pleural fluid is still routinely submitted for diagnosis. In India, smear microscopy is commonly used for screening purposes. It is cheap and rapid, but less sensitive due to the paucibacillary nature of pleural fluid and cannot differentiate M.tuberculosis from other non tuberculous mycobacterium species. The sensitivity of microscopy for pleural fluid and pleural biopsy were very less around $<10 \%$ and $<$ $50 \%$ respectively (L $\mathrm{Li}$ et al., 2019). According to S Mirza et al., 2003; smear microscopy of pleural fluid requires $10^{4}$ bacilli/ml and cultures needs 10-100 viable bacilli/ml. Several researchers reported smear positivity ranging from $0 \%$ to $31 \%$ (Makeshkumar et al., 2014; Siddiqui et al., 2013; Shital et al., 2014; Maurya et al., 2012; Kumar et al., 2010; Khosravi et al., 2017; Lee et al., 2015; Maurya et al., 2011). Hence for timely diagnosis, smear microscopy of pleural fluid alone is not dependable and there is a need for better diagnostic techniques like culture and PCR.

Though LJ culture is less sensitive for pleural fluid, researchers have reported positivity ranging from 0\%-70\% (Sharma et al., 2004; Makeshkumar et al., 2014; Siddiqui et al., 2013; Shital et al., 2014). In the present study, we could report only $2.8 \%$ positivity on LJ. Among the 36 pleural fluids processed, two smear positive samples have not grown on LJ. This may be due to the non-uniform distribution of bacilli in the inoculum and could have been erroneously inferred as due to dead bacilli, if liquid culture system were not employed. This underlines the need for inclusion of liquid culture. Gopi et al., suggested bed side inoculation and incorporation of liquid culture system for pleural fluid samples (Gopi et al., 2007). In our study MGIT recorded $16.7 \%$ positivity. Table-1 compares PCR results with smear and culture. Around $25 \%$ culture positivity from pleural fluid was reported in MGIT system (Siddiqui et al., 2013; Goyal et al., 2015). Among the 34 smear negative samples 
processed in the present study $11.8 \%$ isolates have grown in MGIT. Bunger et. al reported MGIT as a better system for detecting MTB in smear negative samples ( $\mathrm{R}$ Bunger et al., 2013).

As reviewed by Gill et al., PCR sensitivity targeting IS6110 region was near 100\% and specificity ranged from $60 \%-90 \%$ (Gill et al., 2013). A sensitivity of $66 \%$ and specificity of $83 \%$ was observed in our study. As a standalone technique, PCR was positive in $25 \%$ of our cases, but when combined with other conventional techniques it was $30.6 \%$. In this study, two culture positive samples were negative by PCR and hence the sensitivity and specificity for PCR was $66 \%$ and $83 \%$ respectively. This decreased sensitivity may be due to undetectable amount of DNA and/or presence of less copies of target IS6110 region. PCR alone was positive in $16.7 \%$ of the 30 smear and culture negative specimens. Since the EPTB specimens are paucibacillary, PCR helps in the detection of smear and culture negative specimens. False positivity is very common in PCR due to carry over contamination, dead bacilli etc. and since nested PCR is carried out in the present study the chances of false positivity is comparatively less. Khosravi et al., reported that the detection of several target genes will increase sensitivity and eliminate false positive and false negative results (AD Khosravi et al., 2017). PCR can be used as an adjunct test for diagnosis of TB pleuritis along with other parameters and correlate with clinical conditions.

Today molecular techniques like Xpert $\mathrm{MTB} / \mathrm{RIF}$ is used for rapid identification of MTB and rifampicin resistance with conditional recommendations for EPTB specimens. However, WHO and CDC recommend Xpert MTB/RIF positive cases to be confirmed by culture and drug susceptibility testing.
To conclude it may be said that smear microscopy and LJ are less sensitive tools for diagnosis of TB pleuritis. MGIT automated system helps in the early isolation, but less sensitive for pleural fluid compared to PCR. Till now TB PCR is not approved by FDA. TB PCR plays a role where conventional techniques remain negative. Hence PCR can be used as an adjunct test along with smear and culture reports and should be correlated with clinical history, radiological evidence, etc. so as to initiate prompt treatment.

\section{Acknowledgement}

The authors express their sincere gratitude to The Chairman, Vice-Chancellor, DeanResearch \& Allied Health Sciences and DeanFaculty of Medicine, Mahatma Gandhi Medical College \& Research Institute, Pondicherry for their support and encouragement.

\section{References}

Bunger, R., V.A. Singh, Avneet, S. Mehta and Pathania, D. 2013. Evaluation of BACTEC Micro MGIT with Lowenstein Jensen Media for Detection of Mycobacteria in Clinically Suspected Patients of Extra Pulmonary Tuberculosis in a Tertiary Care Hospital at Mullana (Ambala). J Med Microb Diagn. 2:123.

Gill, M.K., S. Kukreja and Chhabra, N. 2013. Evaluation of nested polymerase chain reaction for rapid diagnosis of clinically suspected tuberculous pleurisy. J Clin Diagn Res. 7:2456-8.

Gopi, A., S.M. Madhavan, S.K Sharma and Sahn, S.A. 2007. Diagnosis and Treatment of Tuberculous Pleural Effusion in 2006. Chest. 131:880-889.

Goyal, S., B. Malhotra, S. Bhargava and Verma, D. 2015. Utility of MGIT-960 for detection of Mycobacterium tuberculosis in extrapulmonary specimens. Indian Journal of Applied Research. 11:626-628.

James, P., R. Gupta, D.J Christopher and 
Balamugesh, T. 2010. Evaluation of the diagnostic yield and safety of closed pleural biopsy in the diagnosis of pleural effusion. Indian J Tuberc. 57:19-24.

Kandhakumari, G., and Stephen, S. 2015. Extra pulmonary tuberculosis: Rapid identification of Mycobacterium tuberculosis grown in Mycobacterium growth indicator tube 960 and LowensteinJensen media, employing Standard diagnostics Bioline Mycobacterium tuberculosis protein 64 antigen detection kit. Indian J Med Microbiol 33:S122-125.

Khosravi, A.D., A. Alami, H. Meghdadi and Hosseini, A.A. 2017. Identification of Mycobacterium tuberculosis in Clinical Specimens of Patients Suspected of Having Extrapulmonary Tuberculosis by Application of Nested PCR on Five Different Genes. Front Cell Infect Microbiol. 7:3.

Kumar, P., M.K Sen, D.S Chauhan, V.M Katoch, S. Singh, and Prasad, H.K. 2010. Assessment of the N-PCR assay in diagnosis of pleural tuberculosis: Detection of $M$. tuberculosis in pleural fluid and sputum collected in tandem. PLoS One. 5(4):e10220.

Lee, B.H., S.H. Yoon, H.J. Yeo, D.W. Kim, S.E. Lee, W.H. Cho, S.J. Lee, Y.S. Kim and Jeon, D. 2015. Impact of Implementation of an Automated Liquid Culture System on Diagnosis of Tuberculous Pleurisy. J Korean Med Sci. 30:871-5.

Li, L., Y. Wang, R. Zhang, D. Liu, Y. Li, Y. Zhou, J. Song, W. Li and Tian, P. 2019. Diagnostic value of polymerase chain reaction/acid-fast bacilli in conjunction with computed tomography-guided pleural biopsy in tuberculous pleurisy: A diagnostic accuracy study. Medicine (Baltimore). 98(29):e15992.
Makeshkumar, V., R. Madhavan and Narayanan, S. 2014. Polymerase chain reaction targeting insertion sequence for the diagnosis of extrapulmonary tuberculosis. Indian J Med Res. 139:161-166.

Maurya, A.K., S. Kant, R.A Kushwaha, V.L Nag, M. Kumar and Dhole, T.N. 2011. The advantage of using IS6110-PCR vs. BACTEC culture for rapid detection of Mycobacterium tuberculosis from pleural fluid in northern India. Biosci Trends. 5:159-64.

Maurya, A.K., S. Kant, V.L Nag, R. Kushwaha and Dhole, T.N. 2012. Detection of $123 \mathrm{bp}$ fragment of insertion element IS6110 Mycobacterium tuberculosis for diagnosis of extrapulmonary tuberculosis. Indian $\mathbf{J}$ Med Microbiol. 30:182-6.

Mirza, S., I.B. Restrepo, J.B Mccormick and Fisher-hoch, S.P. 2003. Diagnosis of tuberculosis lymphadenitis using polymerase reaction on peripheral blood mononuclear cells. Am J Trop Med Hyg. 69:461-465.

Sharma, S.K., and Mohan, A. 2004. Extrapulmonary Tuberculosis. Indian J Med Res.120:316-353.

Shital, P., H. Gajanan and Rujuta, A. 2014. Role of Nucleic Acid Amplification Tests (NAATs) in Tuberculous Pleural Effusion: Where It Fits In Routine Diagnostic Workup? J Cell Sci Ther. 5:169.

Siddiqui, M.A.M., P.R Anuradha, K. Nagamani and Vishnu, P.H. 2013. Comparison of conventional diagnostic modalities, BACTEC culture with polymerase chain reaction for diagnosis of extra-pulmonary tuberculosis. J Med Allied Sci. 3:53-58.

World Health Organization. Global Tuberculosis Report 2018. Available from: https://www.who.int/tb/publications/global_ report/en/.

\section{How to cite this article:}

Gandhi Kandhakumari and Selvaraj Stephen. 2020. Diagnostic Role of Polymerase Chain Reaction and Inadequacy of Mycobacterium Growth Indicator Tube culture in Tuberculous Pleuritis. Int.J.Curr.Microbiol.App.Sci. 9(03): 308-312.

doi: https://doi.org/10.20546/ijcmas.2020.903.036 